\title{
Role of high ponderomotive energy in laser-induced nonsequential double ionization
}

\author{
T. Shaaran, ${ }^{1}$ N. Camus, ${ }^{1}$ J. Dura, ${ }^{2}$ L. Fechner,${ }^{1}$ A. Thai, ${ }^{2}$ A. Britz, ${ }^{2}$ M. Baudisch, ${ }^{2}$ T. Steinle, ${ }^{2}$ A. Senftleben, ${ }^{3}$ C. D. Schröter,${ }^{1}$ \\ J. Ullrich, ${ }^{1,4}$ T. Pfeifer, ${ }^{1}$ C. H. Keitel, ${ }^{1}$ J. Biegert, ${ }^{2,5}$ K. Z. Hatsagortsyan,,${ }^{1, *}$ and R. Moshammer ${ }^{1, \dagger}$ \\ ${ }^{1}$ Max-Planck-Institute fur Kernphysik, Saupfercheckweg 1, D-69117 Heidelberg, Germany \\ ${ }^{2}$ ICFO-Institut de Cienciés Fotoniques, Mediterranean Technology Park, 08860 Castelldefels (Barcelona), Spain \\ ${ }^{3}$ Institute of Physics, Center for Interdisciplinary Nanostructure Science and Technology (CINSaT), \\ University of Kassel, Heinrich-Plett-Strasse 40, 34132 Kassel, Germany \\ ${ }^{4}$ Physikalisch-Technische Bundesanstalt, Bundesallee 100, 38116 Braunschweig, Germany \\ ${ }^{5}$ ICREA-Institució Catalana de Recerca i Estudis Avançats, 08010 Barcelona, Spain
}

(Received 1 August 2018; published 19 February 2019)

\begin{abstract}
The laser-induced nonsequential double ionization (NSDI) of rare gas atoms in the near and mid-IR laser fields is studied experimentally and theoretically. We investigate electron-electron correlation at high recollision energies, experimentally achieving ponderomotive energies $\left(U_{p}\right)$ above $80 \mathrm{eV}$. The contribution of the two dominant channels of NSDI in the photoelectron momentum distribution, impact, and excitation ionization, are both shown to scale with ponderomotive energy and are well reproduced by theory. Surprisingly, for a large $U_{p}$ in mid-IR fields, a noticeable electron-electron anticorrelation signal emerges at low photoelectron momenta, which cannot be explained by these mechanisms within state-of-the-art theoretical approaches.
\end{abstract}

DOI: 10.1103/PhysRevA.99.023421

\section{INTRODUCTION}

In the context of strong field-matter interaction, nonequential double ionization (NSDI) is a distinguished process where correlation between electrons plays a significant role [1,2]. The electron correlation in NSDI is initiated by laser-induced rescattering $[3,4]$. In this process, the first electron is released into the continuum by tunneling ionization and, subsequently, it is driven back by the field towards the parent ion, recollides with it, and induces the ionization of the second electron [5-7].

For NSDI, the $(e, 2 e)$-like double ionization and recollision-induced excitation with subsequent ionization (RESI) are the two main mechanisms describing the release of the second electron from the bound state. In the $(e, 2 e)$ scenario, the second electron is directly ionized on the impact, whereas in RESI, the second electron is first promoted to an excited bound state before being ionized by the laser field. The kinematically complete study of NSDI is commonly carried out using the so-called correlation plot representing the the longitudinal photoelectron momentum distribution (along the laser polarization) of both electrons [8]. The contributions on the photoelectron momentum distribution (PMD) of these different channels exhibit characteristic scaling properties in their dependence on the laser parameters, such as $U_{p}$ the ponderomotive energy [9], where $U_{p}=E_{0}^{2} /\left(4 \omega^{2}\right)$ with the peak field $E_{0}$ and the frequency $\omega$ (atomic units are used throughout).

In the $(e, 2 e)$ channel, the first electron most probably returns to the core when the electric field is close to zero and the vector potential near its maximum $2 \sqrt{U_{p}}$. As a result, both

\footnotetext{
*karen.hatsagortsyan@mpi-hd.mpg.de

†robert.moshammer@mpi-hd.mpg.de
}

electrons appear in the continuum at the same time, acquiring a large momentum from the laser (up to $2 \sqrt{U_{p}}$ ) and are emitted in the same direction. These events, commonly called "correlated" electrons, populate quadrants I $\left(p_{1 \|}, p_{2 \|}>0\right)$ and III $\left(p_{1 \|}, p_{2 \|}<0\right)$ in the PMD. The two released electrons further exchange momentum in the continuum, giving rise to a V-shape structure in PMD [10-14].

In RESI, rescattering and excitation also happen most probably at the field crossing. Models, such as the simpleman's neglect the following interaction of the excited state with the laser field and set the moment of ionization to the field maximum. This yields a momentum distribution centered around zero. As a result, electron momentum distributions populate all four quadrants.

Although kinematic complete studies are available for quite some time (for a review see Refs. [3,4]), still there is no unified picture how PMD features evolve with increase of ponderomotive potential. This especially relates to RESI. Several theoretical models have been proposed to explain the general features of RESI [13,15-19], however, no clear conclusion exists about the role of Coulomb effects, depletion of the excited state, and on excitation channels. When considering results obtained using ultrashort pulses, the pattern is largely defined by depletion effects [20-22], contributions of doubly excited states [23], soft recollisions [24], and quantum interferences of different excitation channels [25].

One reason for controversies is that at common near-IR laser wavelengths and intensities the contribution of $(e, 2 e)$ and RESI channels overlap in PMD, which limits the detailed investigation of the underlying processes and comparison between experiment and theory.

To experimentally resolve each of the processes, one option is to vary the ponderomotive energy since these processes have different scaling laws with $U_{p}$. In addition, $U_{p}$ directly influences the kinetic energy of the recolliding electron and 
with it the cross sections for excitation or ionization on impact. These cross sections depend as well on the target ion and its ionization potential $I_{p}[26,27]$. For example, for argon, RESI is dominant over a large range of recollision energy (up to $\left.5 I_{p}\right)$ whereas for neon $(e, 2 e)$, it is dominant starting at $1.5 I_{p}$. These considerations have driven the interpretation of the different spectra observed for the two species and the interest in studying both of them.

The intensity and the wavelength are the two parameters to change $U_{p}$. Going to large intensity is often limited by the appearance of the competing sequential double ionization and, therefore, new perspectives to study NSDI in a large region of ponderomotive energies were opened by recent advances in laser technique in the mid-IR domain [28]. The first experiments with mid-IR lasers in which the ion recoil momentum distributions were measured in NSDI [28-30] indicate the diminishing role of the RESI channel at high ponderomotive energies. In addition, the classical analysis of NSDI in the mid-IR domain [31] indicated modification of the recollision dynamics with respect to the short-wavelength case.

In this paper, we carry out a comparative study of NSDI for argon and neon atoms. Argon atoms are exposed to multicycle laser pulses with wavelengths of 800, 1300, and $3100 \mathrm{~nm}$ and with intensities corresponding to the ponderomotive energy $U_{p}=0.328, U_{p}=0.867$, and $U_{p}=2.957$ a.u., respectively. For neon atoms, results are obtained for 800- and 1300-nm laser pulses with intensities corresponding to $U_{p}=0.656$ and $U_{p}=1.502$ a.u., respectively.

We demonstrate that for argon both $(e, 2 e)$ and RESI mechanisms contribute with the overwhelming role of the $(e, 2 e)$ channel at higher $U_{p}$, whereas for neon $(e, 2 e)$ is the main mechanism for NSDI in all investigated $U_{p}$ values. For argon at the largest $U_{p}$, a noticeable anticorrelation signal emerges at low photoelectron momenta which cannot be explained through the common RESI formalism.

\section{EXPERIMENTAL METHODS}

The experiments at 800 and $1300 \mathrm{~nm}$ were performed with a Ti:sapphire laser system running at $3 \mathrm{kHz}$ and a subsequent optical parametric amplifier (TOPAS) for wavelength conversion. We used either the 25-fs pulses (FWHM) at the fundamental wavelength $(800 \mathrm{~nm})$ or the approximate $60-\mathrm{fs}$ (FWHM) long pulses at a wavelength of $1300 \mathrm{~nm}$ generated with the TOPAS. The experimental results for $3100 \mathrm{~nm}$ were obtained with an optical parametric chirped pulse amplification system [32] operated at a repetition rate of $160 \mathrm{kHz}$ and with pulse durations of $60 \mathrm{fs}$ (FWHM). The laser beam was focused onto a spot of $\approx 10 \mu \mathrm{m}$ diameter into the center of a reaction microscope [33] where a supersonic gas beam was traversing the ultra-high-vacuum chamber (residual gas pressure of $<10^{10}$ mbars). The electrons and ions created in the laser focus were extracted and guided by weak homogenous electric and magnetic fields onto time- and position-sensitive detectors. Their momentum vectors were recorded with a momentum resolution of $\Delta p \approx 0.1$ au. In all cases, dependent on the chosen wavelength and the laser intensity, the gas-jet density was adjusted such that less than one ionization event occurred per laser shot in order to assure a correct assignment of the ion and the coincidently detected electron(s). Thus, the dominant single ionization sets an upper limit for the effective count rate of double ionization which calls for rather long acquisition times (up to 3 days), in particular, for the low repetition rate laser $(3 \mathrm{kHz})$. The stability of all experimentally relevant parameters was maintained throughout the measurements. The limited detector efficiencies reduce the true count rate further. For a true double-ionization event, the two electrons and the doubly charged ion must be detected in coincidence, and their sum momentum must be equal to zero. However, with detector efficiencies of $\approx 50 \%$, this happens for only $\approx 12.5 \%$ of all double-ionization events. In addition, due to the dead time, two electrons that hit the detector within $15 \mathrm{~ns}$ will not be recorded as separate particles. To circumvent these limitations, we in addition accept events where only one electron was detected in coincidence with the double-charged ion, whereas the momentum of the missing second electron was reconstructed by using momentum conservation. This leads to a slight increase in false coincidences which were kept below $20 \%$ for all cases.

\section{THEORETICAL MODEL}

For a theoretical interpretation of the experimental results, strong-field approximation (SFA) is employed. The external laser field is approximated by a monochromatic wave. The $(e, 2 e)$ channel of NSDI is described by the second-order SFA amplitude following the treatment of Ref. [11]. The effect of the ion Coulomb field for the first electron excursion is neglected describing the state $|\mathbf{k}\rangle$ by a plane wave. However, the Coulomb repulsion of the two-electron continuum, which is responsible for the $\mathrm{V}$-structure features, are taken into account employing the exact solution of the Schrödinger equation of two electrons interacting with a laser field [34],

$$
\begin{aligned}
\left|\mathbf{p}_{1}, \mathbf{p}_{2}\right\rangle= & \left|\psi_{\mathbf{p}_{1}}^{V}\right\rangle\left|\psi_{\mathbf{p}_{2}}^{V}\right\rangle \\
& \times \Gamma(1+i / \xi)_{1} F_{1}[-i / \xi ; 1 ; i(p r-\mathbf{p} \cdot \mathbf{r})]
\end{aligned}
$$

where $\mathbf{p}=\left(\mathbf{p}_{1}-\mathbf{p}_{2}\right) / 2, \xi=\left|\mathbf{p}_{1}-\mathbf{p}_{2}\right|, \Gamma(z)$ is the $\Gamma$ function, ${ }_{1} F_{1}[x ; 1 ; z]$ is the confluent hypergeometric function, and $\left|\psi_{\mathbf{p}_{n}}^{V}\right\rangle$ is the Volkov wave function. In the transition amplitude, the electron-electron interaction, which is responsible for dislodging the second electron at the rescattering, is described by the effective Yukawa potential $V_{12}=\delta\left(\mathbf{r}_{2}\right) e^{-\alpha\left|\mathbf{r}_{1}-\mathbf{r}_{2}\right|} /\left|\mathbf{r}_{1}-\mathbf{r}_{2}\right|$ with the effective screening factor of $\alpha \sim 2 E_{2 g}$ and $E_{2 g}$ is the ionization potential of the second electron. For a multielectron system, the accurate accounting for this interaction is a difficult task $[14,35,36]$, and this potential accounts for the screening of the ionic core by the other electrons. Additionally, the localization of the second electron during the interaction is described via the $\delta\left(\mathbf{r}_{2}\right)$ function. The experimental PMD is more closely reproduced when the localization is taken into account. The accurate description of the $(e, 2 e)$ channel allows us to extract correctly the RESI signal from the experimental data.

We model RESI by using the SFA transition amplitude given by Eq. (1) of Ref. [15] and account for the Coulomb effect of the ion in the calculation of the excitation amplitude of the residual ion by employing the Coulomb-Born approximation for describing the continuum state of the recolliding 


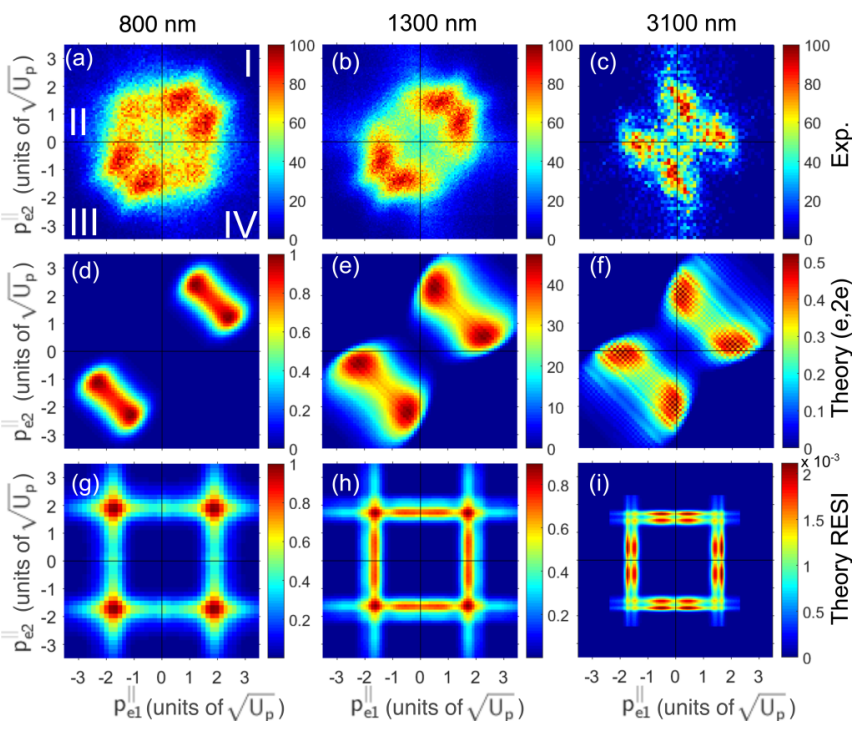

FIG. 1. PMD for argon NSDI: (a)-(c) experiment; (d)-(f) theoretical distributions via the $(e, 2 e)$ channel; $(\mathrm{g})-(\mathrm{i})$ theoretical distributions via the RESI channel. The laser wavelength is $\lambda=800 \mathrm{~nm}$, and the laser intensity is $I=1.5 \times 10^{14} \mathrm{~W} / \mathrm{cm}^{2}\left[U_{p}=0.328\right.$ a.u. $]$ in $(\mathrm{a}, \mathrm{d}$, and $\mathrm{g}), \lambda=1300 \mathrm{~nm}$ and $I=1.5 \times 10^{14} \mathrm{~W} / \mathrm{cm}^{2}\left[U_{p}=\right.$ 0.867 a.u.] in (b, e, and h), $\lambda=3100 \mathrm{~nm}$ and $I=0.9 \times$ $10^{14} \mathrm{~W} / \mathrm{cm}^{2}\left[U_{p}=2.957\right.$ a.u. $]$ in (c, $\mathrm{f}$, and i). The theoretical distributions are without focal volume averaging. The roman labels in (a) correspond to the quadrants referred to in the text.

electron [37]. The latter is very important at the threshold energies, i.e., at energies of the returning electron close to the excitation energy of the target ion, significantly modifying the PMDs for RESI. Furthermore, in the tunnel ionization prefactor, the Coulomb correction part of the AmmosovDelone-Krainov ionization model is included. We consider the variation in the electron binding energy of the atom exposed to the external field due to the ac Stark effect using $E_{n}=E_{0}+\alpha F^{2} / 2$, where $\alpha$ is the static polarizability of the atom. Depletion effects are included by using the empirical model of Ref. [38]. For argon, we consider excitation from $3 p$ to $4 s, 4 p$, and $3 d$ with different angular momenta, which correspond to the channels with the largest cross section [39]. The final distributions are obtained by adding incoherently the PMD of all the individual excitation channels.

\section{RESULTS AND DISCUSSIONS}

\section{A. Argon}

The PMDs of argon are displayed in Fig. 1. The experimental correlation plots are shown in the upper row with increasing ponderomotive energy. In all cases correlated (quadrants I and III) as well as anticorrelated electron (quadrants II and IV) are observed. The middle row and bottom rows represent their corresponding theoretical calculations for $(e, 2 e)$ and RESI mechanisms, respectively. All graphs' axes are shown in units of $\sqrt{U_{p}}$. The calculations for each channel involve different theoretical treatments. Therefore, the independent count scales for each process are relative to the respective maximum at $800 \mathrm{~nm}$.
We can observe both in the experiment and in the theory that the momentum space in the PMDs increases with increasing ponderomotive potential. The $\mathrm{V}$-shape structure in quadrants I and III, characterizing the $(e, 2 e)$ pattern, is shifted towards larger momentum, and the separation of the $\mathrm{V}$ structure wings is increased along with $\sqrt{U_{p}}$ : Both electrons share more asymmetrically their total energy with increasing wavelength. In the calculations, the V-shape structure is slightly wider compared to the experimental findings, which is due to neglecting the interaction of the tunneled and released electrons with the core once they are freed in the laser field. Noticeably, the centers of the correlated momentum spectra in quadrants I and III are at lower momenta than the prediction of the simple man model of $p_{1 \|}=p_{2 \|}= \pm 2 \sqrt{U_{p}}$. This stems from the energy dependence of the impact ionization amplitude. It decreases for higher energy, shifting the center of the PMDs towards lower momenta.

We now focus on the RESI channel and its $U_{p}$ dependence. Our theoretical calculations for RESI, Figs. 1(g)-1(i), show significant signals also in the II and IV quadrants. The RESI signal along the diagonal is due to the ionization of the excited states almost immediately after excitation at the field crossing and long before the electric field reaches its maximum. As a result, the second electron reaches the continuum with momenta larger than zero, very close to $\pm 2 \sqrt{U_{p}}$. This leads to square-shaped electron momentum distributions. The general shapes of the calculated RESI distributions are not as broad as the experiment suggests. This is due to averaging effects (in the focal volume and along the pulse duration) of the intensity present in the experiment, cf. Ref. [36].

The RESI signal decreases as we move towards longer wavelengths in agreement with the previously reported results based on ion momentum spectra [29,30], and it is difficult to recognize in the experimental PMDs in Fig. 1(c). To explore contributions to PMD from other processes than $(e, 2 e)$ (as expected to be mostly induced by RESI), we extract the counts in the II and IV quadrants, within a sector with opening angles of $\Delta \phi=60^{\circ}\left(105^{\circ}<\phi<165^{\circ}\right.$, and $\left.285^{\circ}<\phi<345^{\circ}\right)$ where $\phi$ is the angle of the event $\left(p_{1 \|}, p_{2 \|}\right)$ in the PMD, taking into account that the $(e, 2 e)$ contribution is mostly concentrated in the I and III quadrants. In Fig. 2, we present the distributions of double-ionization events in these segments as a function of their radius $P_{r}=\sqrt{p_{1 \|}^{2}+p_{2 \|}^{2}}$ in the PMDs. The distributions are weighted with $1 / P_{r}$.

Our theory shows that the peaks and FWHM of RESI scales with $\sqrt{U_{p}}$ for all three cases, see Fig. 2(b), which is in qualitative agreement with the experimental results for $\lambda=800$ and $\lambda=1300 \mathrm{~nm}$. At $\lambda=3100 \mathrm{~nm}$, the experimental distribution exhibits a low-momentum peak that resembles the $\lambda=800-\mathrm{nm}$ structure and has a tail in the region of $1<P_{r}<4$, which coincides with the range of the calculated RESI distribution in Fig. 2(b). In addition, the theoretical RESI yields for $\lambda=3100 \mathrm{~nm}$ is about 270 times smaller than for $\lambda=1300 \mathrm{~nm}$, whereas the $(e, 2 e)$ signal is almost the same. This may suggest to attribute the tail of the experimental distribution $\left(1<P_{r}<4\right)$ to the calculated RESI signal.

The comparison between the theory and the experiment shows that there is an anticorrelation momentum distribution at low momenta in the experiment, especially prominent at large ponderomotive energy in mid-IR fields $\left(U_{p}=2.957\right.$, 

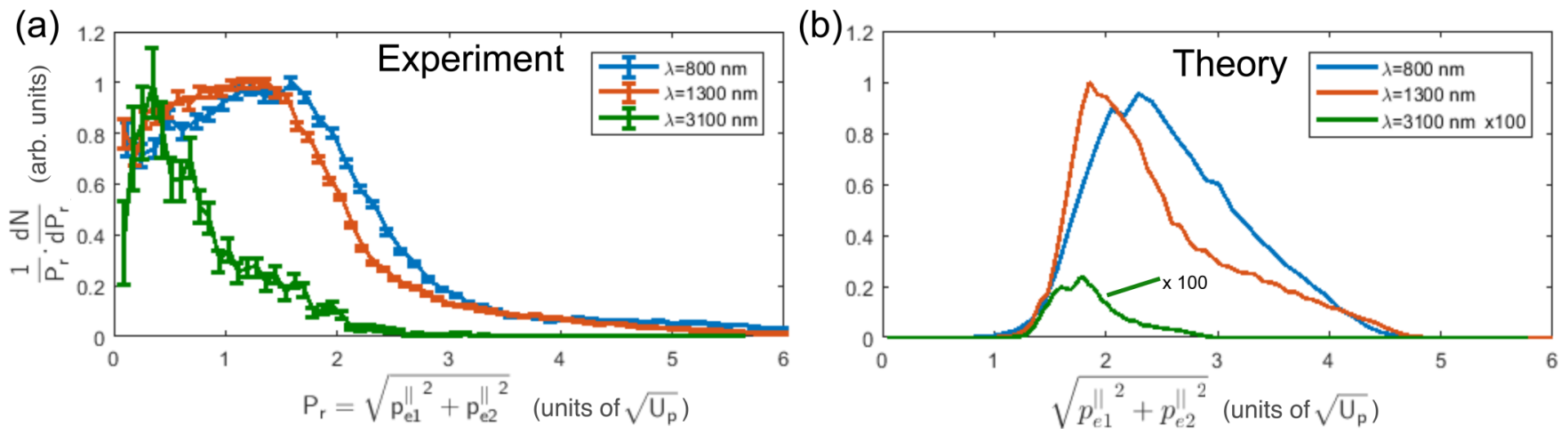

FIG. 2. Photoelectron distribution with respect to $\sqrt{p_{1 \|}^{2}+p_{2 \|}^{2}}$ in quadrants II and IV integrated within the angle $105^{\circ}<\phi<165^{\circ}$, and $285^{\circ}<\phi<345^{\circ}$ normalized to unit phase space: for 800, 1300, and $3100 \mathrm{~nm}$ : (a) experiment, (b) theory.

$\lambda=3100 \mathrm{~nm}$ ) which cannot be explained by the presented RESI theory. In fact, the peak of the anticorrelation signal at $\approx 1.5 \sqrt{U_{p}}$ in the cases of 800 and $1300 \mathrm{~nm}$ is qualitatively reproduced by the theory, whereas the peak of this signal at $3100 \mathrm{~nm}$ in experiment is at much lower energies $\approx 0.3 \sqrt{U_{p}}$ and absent in the theory. The possibility to see sequential double ionization is excluded here since the distribution is not peaking at zero. In the present theory, the excitation takes place at the first return of the electron to the core. There are indications that in mid-IR laser fields the electron recollision current density could be non-negligible at the third return due to Coulomb focusing $[31,40]$. However, this cannot change significantly the leading contribution to RESI. Soft recollisions also may contribute to the anticorrelation signal [24] but not at such low energies (0.08 a.u.) as in Fig. 2. Thus, the low momenta anticorrelation distribution cannot be explained by improvement of the approximations applied within the common RESI model but would require invoking

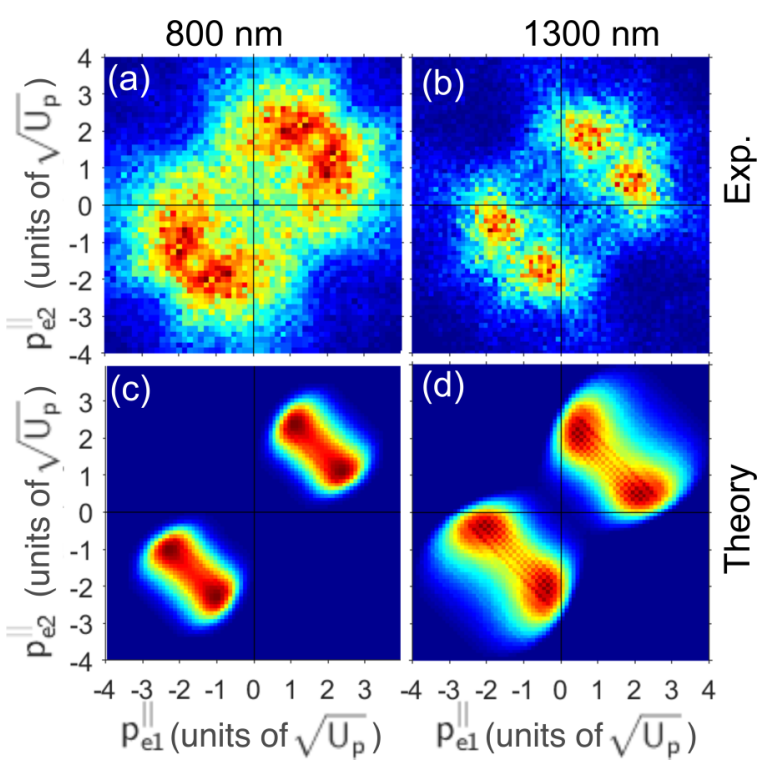

FIG. 3. PMD for neon NSDI: Upper panels: experimental distributions; Bottom panels: theoretical distributions via the $(e, 2 e)$ channel; for two wavelengths: (a) and (c) $800 \mathrm{~nm}$; (b) and (d) 1300 nm. Panels (a) and (c) and (b) and (d) correspond to the laser field with ponderomotive energy $U_{p}=0.656$ a.u. and $U_{p}=1.502$. additional pathways. For instance, one could think of a shakeoff process where both electrons are transiently going into an excited state and then ionized from there close to the same maximum of the field, producing two low-energy electrons.

\section{B. Neon}

The experimental results for neon are shown in Fig. 3. Because of the high intensity needed to tunnel ionization of the first electron, we carried out experiments at 800 and $1300 \mathrm{~nm}$ with a ponderomotive energy of $U_{p}=0.656$ a.u. $(I=3.0 \times$ $\left.10^{14} \mathrm{~W} / \mathrm{cm}^{2}\right)$, and $U_{p}=1.502\left(I=2.6 \times 10^{14} \mathrm{~W} / \mathrm{cm}^{2}\right)$, respectively. For both wavelengths, no significant amount of counts is visible in quadrants II and IV implying that $(e, 2 e)$ is the dominant mechanism. At $800 \mathrm{~nm}$, this is well established $[3,4]$ and explained by the small cross sections for excitation of neon with respect to the direct ionization of the second electron [26,27]. This can also be interpreted by considering that the first excited states in neon have a high energy and the excitation is therefore less favorable than in argon [41]. Our results for $1300 \mathrm{~nm}$ demonstrate that the excitation channel is still negligible even for higher ponderomotive energies, confirming the conclusion of Ref. [30] from ion measurement data. The $(e, 2 e)$ contributions to the correlation plot show the same behavior with respect to $U_{p}$ as in argon. The electron momentum space expands as a function of the ponderomotive energy and the $\mathrm{V}$-shape structure is widening. Again, the good agreement between experiment and theory confirms the validity of our $(e, 2 e)$ model.

\section{CONCLUSION}

We have carried out kinematically complete measurements of laser-induced NSDI of argon and neon in mid-IR laser fields, spanning the ponderomotive energy up to 3 a.u.. For both targets investigated, we have shown that the momentum space of the electron momentum distributions as well as the separation of the V-like structure expand with increasing the ponderomotive energy of the system. The contribution of the RESI channel shows also scaling with $\sqrt{U_{p}}$. However, for high ponderomotive energies, the RESI signal drops down significantly in comparison to the $(e, 2 e)$. In addition, at large $U_{p}$, a well-localized anticorrelation signal emerges at low momenta, which cannot be explained by the RESI mechanism. Our investigation suggests that there should be another 
mechanism, such as the shake-off process, which leads to this localized momentum distribution.

\section{ACKNOWLEDGMENTS}

We acknowledge funding from the Spanish Ministerio de Economía y Competitividad (MINECO) "Severo Ochoa"
Programme for Centres of Excellence in R\&D (Grants No. SEV-2015-0522 and No. FIS2017-89536-P); Catalan Agència de Gestió d'Ajuts Universitaris i de Recerca (AGAUR) (Grant No. 2017 SGR 1639); Fundación Cellex Barcelona; CERCA Programme/Generalitat de Catalunya; LaserlabEurope (Grant No. EU-H2020-654148).

T.S. and N.C. contributed equally to this work.
[1] A. L'Huillier, L. A. Lompre, G. Mainfray, and C. Manus, Phys. Rev. Lett. 48, 1814 (1982).

[2] A. l'Huillier, L. A. Lompre, G. Mainfray, and C. Manus, Phys. Rev. A 27, 2503 (1983).

[3] C. Figueira de Morisson Faria and X. Liu, J. Mod. Opt. 58, 1076 (2011).

[4] W. Becker, X. Liu, P. J. Ho, and J. H. Eberly, Rev. Mod. Phys. 84, 1011 (2012).

[5] P. B. Corkum, Phys. Rev. Lett. 71, 1994 (1993).

[6] B. Walker, B. Sheehy, L. F. DiMauro, P. Agostini, K. J. Schafer, and K. C. Kulander, Phys. Rev. Lett. 73, 1227 (1994).

[7] K. C. Kulander, J. Cooper, and K. J. Schafer, Phys. Rev. A 51, 561 (1995).

[8] T. Weber, H. Giessen, M. Weckenbrock, G. Urbasch, A. Staudte, L. Spielberger, O. Jagutzki, V. Mergel, M. Vollmer, and R. Dorner, Nature (London) 405, 658 (2000).

[9] B. Feuerstein, R. Moshammer, D. Fischer, A. Dorn, C. D. Schröter, J. Deipenwisch, J. R. Crespo Lopez-Urrutia, C. Höhr, P. Neumayer, J. Ullrich, H. Rottke, C. Trump, M. Wittmann, G. Korn, and W. Sandner, Phys. Rev. Lett. 87, 043003 (2001).

[10] S. P. Goreslavski and S. V. Popruzhenko, Opt. Express 8, 395 (2001).

[11] C. Figueira de Morisson Faria, H. Schomerus, X. Liu, and W. Becker, Phys. Rev. A 69, 043405 (2004).

[12] A. Rudenko, V. L. B. de Jesus, T. Ergler, K. Zrost, B. Feuerstein, C. D. Schröter, R. Moshammer, and J. Ullrich, Phys. Rev. Lett. 99, 263003 (2007).

[13] Z. Chen, Y. Liang, and C. D. Lin, Phys. Rev. Lett. 104, 253201 (2010).

[14] D. F. Ye, X. Liu, and J. Liu, Phys. Rev. Lett. 101, 233003 (2008).

[15] T. Shaaran, M. T. Nygren, and C. Figueira de Morisson Faria, Phys. Rev. A 81, 063413 (2010).

[16] D. F. Ye and J. Liu, Phys. Rev. A 81, 043402 (2010).

[17] D. I. Bondar, G. L. Yudin, W.-K. Liu, M. Y. Ivanov, and A. D. Bandrauk, Phys. Rev. A 83, 013420 (2011).

[18] A. S. Maxwell and C. Figueira de Morisson Faria, Phys. Rev. A 92, 023421 (2015).

[19] S. Dong, X. Chen, J. Zhang, and X. Ren, Phys. Rev. A 93, 053410 (2016).

[20] B. Bergues, M. Kübel, N. G. Johnson, B. Fischer, N. Camus, K. J. Betsch, O. Herrwerth, A. Senftleben, A. M. Sayler, T. Rathje, T. Pfeifer, I. Ben-Itzhak, R. R. Jones, G. G. Paulus, F. Krausz, R. Moshammer, J. Ullrich, and M. F. Kling, Nat. Commun. 3, 813 (2012).

[21] M. Kübel, K. J. Betsch, N. G. Kling, A. S. Alnaser, J. Schmidt, U. Kleineberg, Y. Deng, I. Ben-Itzhak, G. G. Paulus, T. Pfeifer, J. Ullrich, R. Moshammer, M. F. Kling, and B. Bergues, New J. Phys. 16, 033008 (2014).
[22] M. Kübel, C. Burger, N. G. Kling, T. Pischke, L. Beaufore, I. Ben-Itzhak, G. G. Paulus, J. Ullrich, T. Pfeifer, R. Moshammer, M. F. Kling, and B. Bergues, Phys. Rev. A 93, 053422 (2016).

[23] N. Camus, B. Fischer, M. Kremer, V. Sharma, A. Rudenko, B. Bergues, M. Kübel, N. G. Johnson, M. F. Kling, T. Pfeifer, J. Ullrich, and R. Moshammer, Phys. Rev. Lett. 108, 073003 (2012).

[24] A. Chen, M. Kübel, B. Bergues, M. F. Kling, and A. Emmanouilidou, Sci. Rep. 7, 7488 (2017).

[25] A. S. Maxwell and C. Figueira de Morrison Faria, Phys. Rev. Lett. 116, 143001 (2016).

[26] V. L. B. de Jesus, B. Feuerstein, K. Zrost, D. Fischer, A. Rudenko, F. Afaneh, C. D. Schröter, R. Moshammer, and J. Ullrich, J. Phys. B: At. Mol. Opt. Phys. 37, L161 (2004).

[27] Z. Chen, Y. Liang, D. H. Madison, and C. D. Lin, Phys. Rev. A 84, 023414 (2011).

[28] B. Wolter, M. G. Pullen, M. Baudisch, M. Sclafani, M. Hemmer, A. Senftleben, C. D. Schröter, J. Ullrich, R. Moshammer, and J. Biegert, Phys. Rev. X 5, 021034 (2015).

[29] A. S. Alnaser, D. Comtois, A. T. Hasan, D. M. Villeneuve, J.-C. Kieffer, and I. V. Litvinyuk, J. Phys. B: At. Mol. Opt. Phys. 41, 031001 (2008).

[30] O. Herrwerth, A. Rudenko, M. Kremer, V. L. B. de Jesus, B. Fischer, G. Gademann, K. Simeonidis, A. Achtelik, T. Ergler, B. Feuerstein, C. D. Schröter, R. Moshammer, and J. Ullrich, New J. Phys. 10, 025007 (2008).

[31] C. Huang, M. Zhong, and Z. Wu, Opt. Express 24, 28361 (2016).

[32] A. Thai, M. Hemmer, P. K. Bates, O. Chalus, and J. Biegert, Opt. Lett. 36, 3918 (2011).

[33] J. Ullrich, R. Moshammer, A. Dorn, R. Dörner, L. P. H. Schmidt, and H. Schmidt-Böcking, Rep. Prog. Phys. 66, 1463 (2003).

[34] F. H. M. Faisal, Phys. Lett. A 187, 180 (1994).

[35] C. Figueira de Morisson Faria, X. Liu, W. Becker, and H. Schomerus, Phys. Rev. A 69, 021402 (2004).

[36] Z. Chen, Y. Liang, and C. D. Lin, Phys. Rev. A 82, 063417 (2010).

[37] T. Shaaran, K. Z. Hatsagortsyan, and C. H. Keitel, Phys. Rev. A 98, 023410 (2018).

[38] X. M. Tong and C. D. Lin, J. Phys. B: At. Mol. Opt. Phys. 38, 2593 (2005).

[39] I. P. Zapesochnyi, A. I. Imre, and Y. N. Semenyuk, Sov. Phys. JETP 72, 400 (1991) .

[40] K. Sasaki, X. M. Tong, and N. Toshima, J. Phys. B: At. Mol. Opt. Phys. 42, 165603 (2009).

[41] Y. Liu, D. Ye, J. Liu, A. Rudenko, S. Tschuch, M. Dürr, M. Siegel, U. Morgner, Q. Gong, R. Moshammer, and J. Ullrich, Phys. Rev. Lett. 104, 173002 (2010). 